\title{
Alpha amylase as a salivary biomarker of acute stress of venepuncture from periodic medical examinations
}

\author{
David Koh ${ }^{1,2 *}$, Vivian $\mathbf{N g}^{2}$ and Lin Naing ${ }^{1}$ \\ 1 PAPRSB Institute of Health Sciences, Universiti Brunei Darussalam, Gadong, Brunei Darussalam \\ ${ }^{2}$ SSH School of Public Health, National University of Singapore, Singapore, Singapore
}

\section{Edited by:}

Jamal Hisham Hashim, United

Nations University, Malaysia

Reviewed by:

Kyoung-Mu Lee, Korean National Open University, South Korea

Siti Munira Yasin, Universiti Teknologi

MARA, Malaysia

\section{*Correspondence:}

David Koh, PAPRSB Institute of Health Sciences, Universiti Brunei Darussalam, Jalan Tungku Link,

Gadong BE 1410, Brunei Darussalam e-mail:david.koh@ubd.edu.bn
Periodic occupational health examinations often require venepuncture. Acute psychological and physical stressors during such procedure result in sympathetic stimulation and increased salivary protein secretion, including salivary $\alpha$-amylase (SAA). We studied SAA response to venepuncture during such examination. Fifty-eight healthy males undergoing periodic medical examination reported perceived stress level (PSL) scores (on a five-point scale) and provided passive drool saliva samples at 15-min (T1) and 1-min before (T2); and 1min (T3) and 15-min after venepuncture (T4). A subset of 33 participants available for repeat examination on a control day when there was no venepuncture provided saliva samples at the corresponding times for comparison. Saliva SAA activity levels were analyzed using a SAA assay kit (Salimetrics LLC, USA). Among 58 participants, mean SAA increased from T1 $(89.95 \mathrm{U} / \mathrm{L})$ to T2 $(109.5 \mathrm{U} / \mathrm{L})$ and T3 $(116.9 \mathrm{U} / \mathrm{L})$. SAA remained elevated $15 \mathrm{~min}$ after venepuncture $(121.0 \mathrm{U} / \mathrm{L})$. A positive trend in the difference of SAA between T3 and T1 was noted among subjects with increasing mean PSL scores. T3-T1 values were 0.6 lamong those with PSL $\leq 1, n=24$ ), 11.3 (among those with PSL between 1 and 1.5, $n=18$ ), and 78.9 (among those with PSL $>1.5, n=16$ ). SAA increment over four-time points was significantly higher on the venepuncture compared to the control day $(P=0.021)$. SAA increases in response to the acute stress of venepuncture during a periodic medical examination, and remains elevated $15 \mathrm{~min}$ after the procedure. In comparison, such fluctuations in SAA were not seen on a control day. During venepuncture, increase in SAA from baseline is higher among those who reported greater self-perceived stress during the procedure.

Keywords: salivary biomarker, alpha amylase, venepuncture, acute stress, periodic medical examination

\section{INTRODUCTION}

The use of periodic medical examinations for occupational health surveillance of workers exposed to workplace hazards is a wellestablished practice. Such examinations may be a simple review of symptoms associated with exposure to a specific hazard, but often require measurement of physiological parameters or biological monitoring of biomarkers of exposure and effect (1). The extraction of venous blood for biological monitoring is a common procedure for workers. This procedure will cause psychological stress and pain for most the people regardless of age $(2,3)$.

Previous studies have indicated that salivary $\alpha$-amylase (SAA) levels increase acutely in response to physical (4-6) as well as psychological stressors (7-12). Most of the studies to assess psychological stress were carried out in a laboratory or in quasinaturalistic settings (9). It was suggested that the secretion of SAA responds to stimulation of the sympathetico-adreno-medullary (SAM) system. This is based on the fact that sympathetic stimulation increases salivary protein secretion (10). Salivary flow rate has also been shown to have negligible impact on stress-induced SAA activity (13). Another finding is that SAA levels can also increase in response to pain. In a study conducted by Shirasaki et al. to investigate the correlation between SAA activity with pain intensity, a lumbar epidural block significantly decreased SAA activity in patients with chronic pain (14).
In this study, we examined the acute stress (using SAA as a marker) among workers who were subjected to a periodic medical examination, which required a venepuncture to be performed. We also investigated the association between changes in SAA and subjects' self-reported stress levels during the procedure.

\section{MATERIALS AND METHODS \\ PARTICIPANTS}

The study was conducted in conjunction with the routine sixmonthly statutory medical examinations of a group of workers employed in a factory manufacturing a lead $(\mathrm{Pb})$ based polyvinyl chloride stabilizer located in the western part of Singapore.

Periodic medical examinations of lead exposed workers by a registered designated workplace doctor are required by law in Singapore. The medical examination involves sampling of venous blood for measurement of hemoglobin and lead.

Approval to conduct this study was obtained from the NUS Institutional Review Board (NUS IRB reference code 05-045).

All 58 male workers working in the factory who were exposed to lead were examined during the statutory periodic medical examination over two working days. Two weeks later, we collected the saliva samples on a control day (at the same time of day) from a subset of 33 workers from the originally studied group of 58 workers. These 33 workers were selected on the basis of their availability 
at the workplace on the control day. During the control day, the 33 participants were gathered in a room and occupied with reading newspapers/magazines.

All workers were briefed about the study and gave written informed consent before participation. Inclusion criteria of participants for the study were males who did not suffer from any occupational or non-communicable non-occupational diseases, including pre-existing psychiatric illness. Workers were advised not to smoke, eat, or consume caffeinated drinks $1 \mathrm{~h}$ prior to saliva collection.

\section{SALIVA SAMPLING AND ANALYSIS}

Saliva samples were collected from each subject at 15-min (T1) and 1-min (T2) before venepuncture/control day activities, and 1-min (T3) and 15-min (T4) after venepuncture/control day activities. At the same time points, subjects were asked to rate their perceived stress levels (PSLs) on a 5-point scale (ranging from $1=$ not stressed at all, to $5=$ extremely stressed) prior to saliva collection. Participants were required to spit through a straw into a microcentrifuge tube to obtain a volume of approximately $1 \mathrm{~mL}$ at each time point.

Saliva collection was performed by research personnel who were trained in saliva collection techniques.

Salivary $\alpha$-amylase activity (U/L) in each sample was measured using a kinetic measurement kit (SAA assay kit, Salimetrics LLC, USA).

\section{STATISTICAL ANALYSIS}

Descriptive statistics were used to describe demographic data and SAA levels. Repeated measures ANOVA (RMANOVA) was used to compare four-time points measures of SAA and PSL among 58 participants. RMANOVA was also used to compare the pattern (through four-time points, T1-T4) of SAA levels between venepuncture and control days among the 33 participants. Required assumptions of RMANOVA such as normality, sphericity, and equal-variance, were checked. As the normality and sphericity assumptions were not met in all RMANOVA analyses, log-transformed SAA and PSL data, and the Greenhouse-Geisser correction procedure were used, respectively. Although there was a wide variation of age in the study sample, inclusion of age as a covariate in the RMANOVA model did not change the result and therefore, it was not included in the final model.

Data were analyzed with IBM SPSS Version 20.0 (SPSS Institute, Chicago, IL, USA). All hypothesis tests were two-sided and $P$ values $<0.05$ were considered as statistically significant.

\section{RESULTS}

\section{DEMOGRAPHIC DATA}

All male 58 lead exposed workers who worked in the factory participated in the study. Their ages ranged between 22 and 61 years, with a mean (SD) age of 36.1 (10.7) years. Their duration of employment at this particular workplace ranged from few months to 24 years. The blood lead levels of all the workers were below $50 \mu \mathrm{g} / \mathrm{dL}$.

A subset of 33 male workers [mean (SD) age 36.4 (9.7) years] who were available on a control day 2 weeks later were re-examined while they were reading newspapers.

\section{CHANGE IN PERCEIVED STRESS LEVEL AND SALIVARY $\alpha$-AMYLASE OF 58 WORKERS}

The 58 subjects who underwent the periodic medical examination indicated that venepuncture was not a stressful procedure, with a PSL score of 1 (76\% at T1 to $83 \%$ at T4). More subjects $(40 \%$ at $\mathrm{T} 2$ to $47 \%$ at T3) indicated they were more stressed (PSL of 2 or more) immediately before and after the venepuncture.

The pattern of the mean scores of PSL and mean SAA levels over the four-time points is presented in Figures $\mathbf{1}$ and $\mathbf{2}$.

Table 1 shows the SAA activities in four-time points among the 58 subjects who underwent the periodic medical examination and the venepuncture. Overall, there was a significant change of SAA level $(P=0.001)$ and the significant change was detected between $\mathrm{T} 1$ and $\mathrm{T} 2(P=0.039)$. As there was a variation of age in the sample, age variable was added into the model to control the effect of age. However, there was no significant change in the result.

Table 2 shows the PSL score at four-time points among the 58 subjects who underwent the periodic medical examination and the venepuncture. Overall, there was a significant change of PSL score $(P<0.001)$ and the significant change was detected between $\mathrm{T} 1$ and $\mathrm{T} 2(P=0.018)$ and T3 and T4 $(P<0.001)$.

Although PSL score significantly dropped from T3 to T4, a slight increase of SAA was observed from T3 to T4 (though it was statistically not significant).

A positive trend in the difference of the SAA level between T3 and T1 was noted among subjects categorized by increasing mean PSL scores (Table 3).

\section{COMPARISON OF SALIVARY $\alpha$-AMYLASE LEVELS OF THE 33 WORKERS DURING THE VENEPUNCTURE AND THE CONTROL DAY}

All SAA levels of the 33 workers were positively skewed, which was shown by mean and standard deviation (SD) in Table 4 . Due to

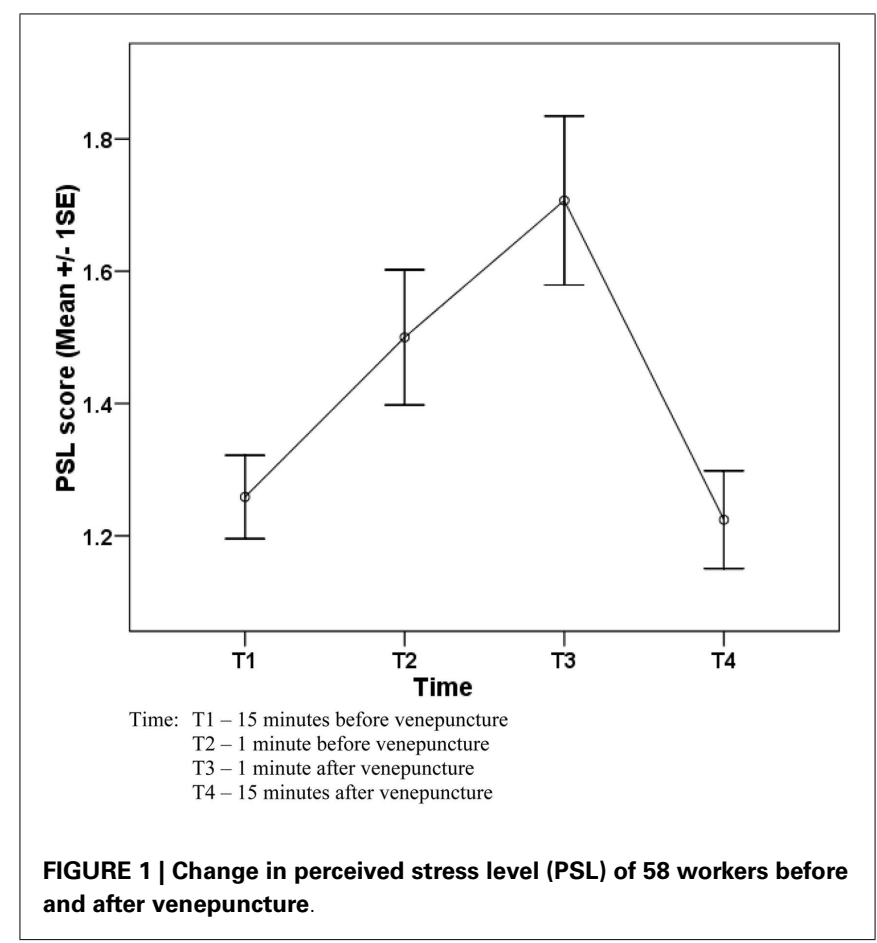




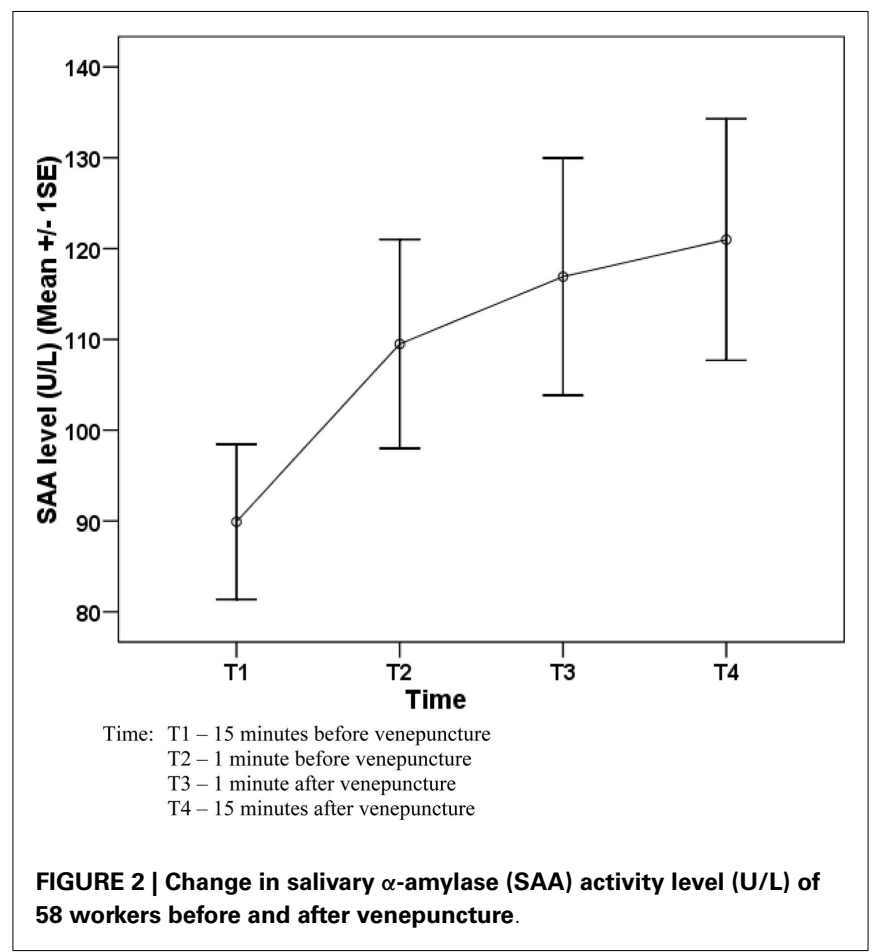

Table 1 | Change of salivary $\alpha$-amylase (SAA) level at four-time measures of 58 workers before and after the venepuncture.

\begin{tabular}{|c|c|c|c|}
\hline \multirow[t]{2}{*}{ Time } & \multicolumn{2}{|c|}{$\begin{array}{l}\text { Salivary } \\
\alpha \text {-amylase (U/L) }\end{array}$} & \multirow[t]{2}{*}{$P$ value } \\
\hline & Mean & SD & \\
\hline \multicolumn{4}{|l|}{ T1 } \\
\hline 15 min before venepuncture & 89.9 & 65.1 & 0.002 (Overall) $^{\mathrm{a}}$ \\
\hline \multicolumn{4}{|l|}{ T2 } \\
\hline $1 \mathrm{~min}$ before venepuncture & 109.5 & 87.7 & $0.036(\mathrm{~T} 1$ vs. T2) \\
\hline \multicolumn{4}{|l|}{ T3 } \\
\hline 1 min after venepuncture & 116.9 & 99.4 & $0.861($ T2 vs. T3) \\
\hline \multicolumn{4}{|l|}{$\mathrm{T} 4$} \\
\hline 15 min after venepuncture & 121.0 & 101.3 & $1.000(\text { T3 vs. T4 })^{\mathrm{b}}$ \\
\hline
\end{tabular}

the skewness of residuals in using RMANOVA in comparing two patterns (venepuncture and control days), log-transformed data were used for the comparison.

Comparison of the pattern of log-transformed SAA levels between venepuncture and control days is presented in Table 3.

The comparison of pattern (T1-T4) was statistically significant $(P=0.021)$ although all the rest in between patterns (T1-T2), (T2-T3), and (T3-T4) were statistically not significant between venepuncture and control days (Figure 3). As there was a wide
Table 2 | Change of perceived stress level (PSL) at four-time measures of 58 workers.

\begin{tabular}{|c|c|c|c|}
\hline \multirow[t]{2}{*}{ Time } & \multicolumn{2}{|c|}{$\begin{array}{l}\text { Perceived } \\
\text { stress level }\end{array}$} & \multirow[t]{2}{*}{$P$ value } \\
\hline & Mean & SD & \\
\hline \multicolumn{4}{|l|}{ T1 } \\
\hline 15 min before venepuncture & 1.3 & 0.48 & $<0.001\left(\right.$ Overall) ${ }^{\mathrm{a}}$ \\
\hline \multicolumn{4}{|l|}{ T2 } \\
\hline $1 \mathrm{~min}$ before venepuncture & 1.5 & 0.78 & $0.054(\mathrm{~T} 1 \mathrm{vs} . \mathrm{T} 2)^{\mathrm{b}}$ \\
\hline \multicolumn{4}{|l|}{ T3 } \\
\hline $1 \mathrm{~min}$ after venepuncture & 1.7 & 0.97 & $0.051($ T2 vs. T3) \\
\hline \multicolumn{4}{|l|}{ T4 } \\
\hline 15 min after venepuncture & 1.2 & 0.56 & $<0.001($ T3 vs. T4) \\
\hline
\end{tabular}

a Repeated measures ANOVA for four-time measures with log transformation (with Greenhouse-Geisser's epsilon correction) ( $F=10.63 ; d f=3 ; 115)$.

${ }^{b}$ Post hoc three multiple comparisons with Bonferroni's procedure.

Table 3 | Change in SAA (T3-T1) among participants categorized by mean PSL score.

\begin{tabular}{lrrrrr}
\hline Mean PSL score (T1-T4) & $\boldsymbol{n}$ & \multicolumn{4}{c}{ SAA change $^{\mathrm{a}}$ (T3-T1) (U/L) } \\
\cline { 3 - 6 } & & Mean & (SD) & Median & (IOR) \\
\hline 1.00 & 24 & 0.6 & 41.2 & 1.6 & 59.5 \\
$1.01-1.50$ & 18 & 11.3 & 50.4 & 1.8 & 32.2 \\
$1.51+$ & 16 & 78.9 & 113.2 & 29.5 & 179.1 \\
Total & 58 & 27.0 & 75.7 & 5.3 & 57.1 \\
& & & & &
\end{tabular}

a Minimum and maximum values were -69 and 307 , respectively.

Table 4 | Comparison of pattern of salivary $\alpha$-amylase (SAA) of 33 workers on venepuncture and control days.

\begin{tabular}{|c|c|c|c|c|c|}
\hline \multirow[t]{3}{*}{ Time } & \multicolumn{4}{|c|}{ SAA level (U/L) } & \multirow{3}{*}{$\begin{array}{c}\text { RMANOVA }^{\mathrm{a}} \\
\text { F Stat, (df), [ } P \text { value] }\end{array}$} \\
\hline & \multicolumn{2}{|c|}{ Venepuncture } & \multicolumn{2}{|c|}{ Control day } & \\
\hline & Mean & SD & Mean & SD & \\
\hline$\lceil 1$ & 75.5 & 51.73 & 95.9 & 56.68 & $5.92,(1 ; 32),[0.021]^{b}$ \\
\hline T2 & 92.1 & 72.47 & 99.4 & 65.02 & $3.23,(1 ; 32),[0.082]^{c}$ \\
\hline$\Gamma 3$ & 94.0 & 81.00 & 94.2 & 66.49 & $1.15,(1 ; 32),[0.291]^{d}$ \\
\hline$\lceil 4$ & 97.2 & 77.03 & 98.6 & 66.34 & $0.43,(1 ; 32),[0.516]^{\mathrm{e}}$ \\
\hline
\end{tabular}

${ }^{a}$ Repeated measures ANOVA using log-transformed SAA data.

${ }^{b}$ Comparing pattern $(T 1, T 4)$.

${ }^{\circ}$ Comparing pattern $(T 1, T 2)$.

${ }^{d}$ Comparing pattern $(T 2, T 3)$.

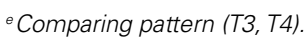

T1, 15 min before venepuncture or control day reading activity.

T2, 1 min before venepuncture or control day reading activity.

T3, 1 min after venepuncture or control day reading activity.

T4, 15 min after venepuncture or control day reading activity. 


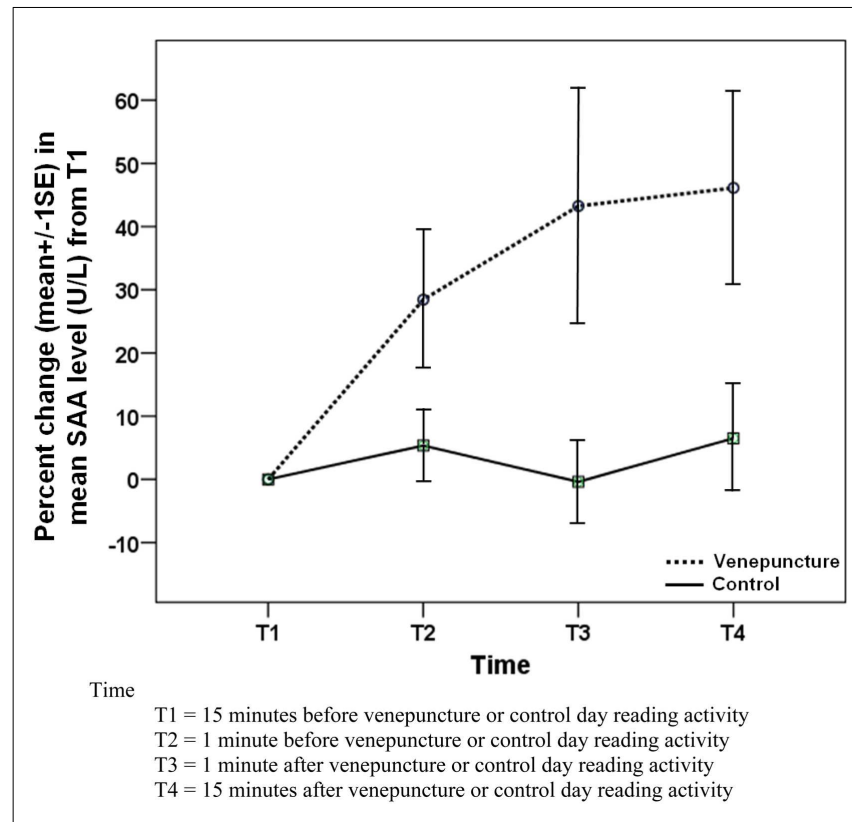

FIGURE 3 | Comparison of change in SAA level between venepuncture and control days among 33 subjects

variation of age in the sample, age variable was added into the model to control the effect of age. However, there was no significant change in the result.

In view of the statistically negative results, a power calculation was done for the sample size of 33. To detect the difference of 20 units of SAA level (if we consider that 20 units difference of change in SAA between two study groups is an important finding to detect), the power of the study was $26.2 \%$, for an alpha of 0.05 . To achieve a study with $80 \%$ power, we would require 142 participants. The within-group largest SD (T1-T4) of SAA was $60 \mathrm{U} / \mathrm{L}$, estimated from the data for the power calculation.

\section{DISCUSSION}

Amylase is a calcium-containing metalloenzyme, which has a number of isoenzymes. In saliva, it is produced by serous acinar cells of the parotid and submandibular salivary glands, and accounts for $10-20 \%$ of total salivary proteins. The parotid glands produce $80 \%$ of all SAA. An important function of SAA is to hydrolyze $\alpha-1,4$ linkages of starch to glucose and maltose. This property allows SAA quantification by measuring its activity in enzyme units per milliliter, where an enzyme unit is the amount of enzyme that catalyzes conversion of $1 \mu \mathrm{mol}$ of substrate per minute. SAA also contributes to oral cavity mucosal immunity by inhibiting adherence and growth of bacteria.

Salivary glands are innervated by both sympathetic and parasympathetic nerves, and increase in SAA is a reflection of autonomic nervous system activity. Sympathetic nerve stimulation via norepinephrine increases total salivary protein concentration, while parasympathetic cholinergic stimulation increases salivary flow rate. As such, SAA activity increases with physical stressors, pain, and psychological stress. Most of the research on
SAA took place in experimental laboratories or quasi-naturalistic settings, but field studies e.g., of acute anticipatory stress and pain due to venepuncture, also show increase and continued elevation of SAA.

Among 58 participants, SAA increased from T1 (mean 89.95 U/L) to T2 (109.5 U/L) and T3 (116.9 U/L). SAA remained elevated $15 \mathrm{~min}$ after venepuncture $(121.0 \mathrm{U} / \mathrm{L})$. The results indicate a significant elevation of SAA from $15 \mathrm{~min}$ before venepuncture to $1 \mathrm{~min}$ before venepuncture. For many people, venepuncture is a painful procedure, which can induce certain level of psychological stress, and it is not surprising to note that the levels of SAA begin to increase even before the subjects underwent venepuncture. This may be due to the stress of anticipation of the painful procedure. It has been reported the psychological stress activates the SAM system $(4,10)$ and results in increased SAA release.

The means SAA levels remained elevated $15 \mathrm{~min}$ after the venepuncture. In comparison, a study of subjects' response to the "Trier Social Stress Test (TSST)" (a psychosocial stress test), showed that SAA levels decreased to the control session level after $20 \mathrm{~min}$ (11). Another study conducted by Gordis and colleagues on adolescents' response to TSST, showed saliva $\alpha$-amylase levels returning to baseline $10 \mathrm{~min}$ post-stressor (7).

Our findings may be due to the study subjects still experiencing residual pain after the venepuncture. Continuing painful stimuli may also activate the SAM system $(15,16)$. Shirasaki et al. (14) suggested that SAA activity may be a good index for the objective assessment of pain intensity and may reflect activity of the SAM system during pain induced stress.

Most of the subjects have been working in the factory for several years; with venepuncture being part of the six-monthly medical examinations. Most of the subjects indicated that venepuncture was not a stressful procedure to them. However, there was still a positive increase in SAA (percentage change of SAA compared to T1) on the venepuncture day. In contrast, the SAA levels were remained constant throughout the four-time points on the control day.

In another study (9), 24 healthy adults were exposed to the TSST - a standardized psychosocial stress test and a control (rest) condition on separate days. SAA levels showed a marked increase during the stress day but showed no significant changes during the control day. This showed that a psychological stressor can also increase SAA levels.

In the subjects undergoing venepuncture during a periodic medical examination, it is probable that both psychological as well as physical stressors contribute to the increase in SAA.

During the venepuncture, the increase in SAA from baseline is higher among those who reported greater self-perceived stress during the procedure. This is indicated in the positive trend in the difference of SAA between T3 and T1 among subjects with increasing PSL scores. T3-T1 values were 0.6 (among low PSL group $n=24$ ), 11.3 (among moderate PSL group $n=18$ ), and 78.9 (among high PSL group $n=16$ ). This suggests that perceived stress can modify the change in SAA.

Furthermore, similar fluctuations in SAA were not seen on the control day over the same 30-min period. Although the 
baseline values of SAA were higher on the control day, SAA relative increment over four-time points was significantly higher on the venepuncture compared to the control day $(P=0.021)$. This suggests that venepuncture does cause a real increase in SAA.

There were some limitations of the study. The saliva samples were collected at 15-min (T1) and 1-min (T2) before venepuncture/control day activities, and 1-min (T3) and 15-min (T4) after that. Considering that the levels of SAA were still elevated after 15 min after venepuncture, additional time points for observation beyond $15 \mathrm{~min}$ should have been included in the study design. However, the workers had to go back to work after the medical examination, and the management was not willing to allow them to spend extra time for the study. Besides age, gender, and psychiatric illness, which were considered in this study, other covariates e.g., personality or individual psychological makeup, might confound the results of this study and need to be considered.

The sample size was not calculated prior to this study, as the maximum study size possible, comprising the entire lead exposed male population of the factory was studied.

\section{CONCLUSION}

Salivary $\alpha$-amylase increases in response to the acute stress of venepuncture during a periodic medical examination, and remains elevated $15 \mathrm{~min}$ after the procedure. Such fluctuations were not seen on a control day. During venepuncture, increase in SAA from baseline is higher among those who reported greater self-perceived stress during the procedure.

Further research is required in this area, e.g., studies with larger sample sizes and which include additional observations for time points beyond $15 \mathrm{~min}$ after the venepuncture, and to examine possible interactions between psychological and physical stressors on SAA levels.

\section{ACKNOWLEDGMENTS}

We would like to thank all participants of the study.

\section{REFERENCES}

1. Koh D, Aw TC. Surveillance in occupational health. Occup Environ Med (2003) 60:705-10. doi:10.1136/oem.60.9.705

2. Meeran K, Hattersley A, Mould G, Bloom SR. Venepuncture causes rapid rise in plasma ACTH. Br J Clin Pract (1993) 47:246-7.

3. Tak JH, van Bon WH. Pain- and distress-reducing interventions for venepuncture in children. Child Care Health Dev (2006) 32:257-68. doi:10.1111/j.13652214.2006.00578.x

4. Chatterton RT Jr, Vogelsong KM, Lu YC, Ellman AB, Hudgens GA. Salivary alpha-amylase as a measure of endogenous adrenergic activity. Clin Physiol (1996) 16:433-48. doi:10.1111/j.1475-097X.1996.tb00731.x
5. Kivlighan KT, Granger DA. Salivary alpha-amylase response to competition: relation to gender, previous experience, and attitudes. Psychoneuroendocrinology (2006) 31:703-14. doi:10.1016/j.psyneuen.2006.01.007

6. Walsh NP, Blannin AK, Clark AM, Cook L, Robson PJ, Gleeson M. The effects of high-intensity intermittent exercise on saliva IgA, total protein and alphaamylase. J Sports Sci (1999) 17:129-34. doi:10.1080/026404199366226

7. Gordis EB, Granger DA, Susman EJ, Trickett PK. Asymmetry between salivary cortisol and alpha-amylase reactivity to stress: relation to aggressive behavior in adolescents. Psychoneuroendocrinology (2006) 31:976-87. doi:10.1016/j. psyneuen.2006.05.010

8. Nater UM, La Marca R, Florin L, Moses A, Langhans W, Koller MM, et al. Stress-induced changes in human salivary alpha-amylase activity - associations with adrenergic activity. Psychoneuroendocrinology (2006) 31:49-58. doi:10.1016/j.psyneuen.2005.05.010

9. Nater UM, Rohleder N, Gaab J, Berger S, Jud A, Kirschbaum C, et al. Human salivary alpha-amylase reactivity in a psychosocial stress paradigm. Int J Psychophysiol (2005) 55:333-42. doi:10.1016/j.ijpsycho.2004.09.009

10. Rohleder N, Nater UM, Wolf JM, Ehlert U, Kirschbaum C. Psychosocial stressinduced activation of salivary alpha-amylase: an indicator of sympathetic activity? Ann N Y Acad Sci (2004) 1032:258-63. doi:10.1196/annals.1314.033

11. Rohleder N, Wolf JM, Herpfer I, Fiebich BL, Kirschbaum C, Lieb K. No response of plasma substance $\mathrm{P}$, but delayed increase of interleukin-1 receptor antagonist to acute psychosocial stress. Life Sci (2006) 78:3082-9. doi:10.1016/ j.lfs.2005.12.016

12. van Stegeren A, Rohleder N, Everaerd W, Wolf OT. Salivary alpha amylase as marker for adrenergic activity during stress: effect of beta-blockade. Psychoneuroendocrinology (2006) 31:137-41. doi:10.1016/j.psyneuen.2005.05.012

13. Rohleder N, Wolf JM, Maldonado EF, Kirschbaum C. The psychosocial stressinduced increase in salivary alpha-amylase is independent of saliva flow rate. Psychophysiology (2006) 43:645-52. doi:10.1111/j.1469-8986.2006.00457.x

14. Shirasaki S, Fujii H, Takahashi M, Sato T, Ebina M, Noto Y, et al. Correlation between salivary alpha-amylase activity and pain scale in patients with chronic pain. Reg Anesth Pain Med (2007) 32:120-3. doi:10.1016/j.rapm.2006.11.008

15. Drummond PD. Involvement of the sympathetic nervous system in complex regional pain syndrome. Int J Low Extrem Wounds (2004) 3:35-42. doi: $10.1177 / 1534734604263365$

16. Janig W. The sympathetic nervous system in pain. Eur J Anaesthesiol Suppl (1995) 10:53-60.

Conflict of Interest Statement: The authors declare that the research was conducted in the absence of any commercial or financial relationships that could be construed as a potential conflict of interest.

Received: 24 June 2014; accepted: 04 August 2014; published online: 26 August 2014. Citation: Koh D, Ng V and Naing L (2014) Alpha amylase as a salivary biomarker of acute stress of venepuncture from periodic medical examinations. Front. Public Health 2:121. doi: 10.3389/fpubh.2014.00121

This article was submitted to Occupational Health and Safety, a section of the journal Frontiers in Public Health.

Copyright $\odot 2014 \mathrm{Koh}, \mathrm{Ng}$ and Naing. This is an open-access article distributed under the terms of the Creative Commons Attribution License (CC BY). The use, distribution or reproduction in other forums is permitted, provided the original author (s) or licensor are credited and that the original publication in this journal is cited, in accordance with accepted academic practice. No use, distribution or reproduction is permitted which does not comply with these terms. 